\title{
ON OPTIMAL REINSURANCE
}

\author{
H. G. VERBEEK \\ Amsterdam
}

\section{Introduction}

In this paper an attempt is made to find an answer to the question, "What is the most advantageous size for the retention limit of a risk portfolio, given the fact that a certain stability requirement is to be satisfied?"

This problem will be approached from the viewpoint of an insurer who wishes to obtain a certain degree of stability at lowest cost.

It is assumed that in his choice of reinsurance methods, the insurer restricts himself to either a surplus treaty, a stop loss treaty or a combination of both these types.

Moreover it is assumed that "stability" can be adequately measured by the variance of the risks retained for own account.

We start to consider a reinsurance policy based on the surplus system where the amount of risk in excess of a retention limit $u$ is ceded.

By thus limiting the potential loss on each risk individually, the variance is kept at a certain level, but at the expense of an amount of premium payable to a reinsurer.

The insurer could, of course, reduce the reinsurance cost by increasing his retention but he then is bound to incur a higher variance in his portfolio, which would mean a loss of stability.

One might ask, however, whether a suitably chosen stop loss coverage could bring the variance down again to the proper level at lesser cost than the profit obtained by taking a higher retention. A reduction in reinsurance cost would then have been effected.

The question leads to an optimization problem, which in a more general setting, has been discussed by K. Borch.*

*) K. Borch: "An Attempt to Determine the Optimum Amount of Stop Loss Reinsurance":

XVIth International Congress of Actuaries, Brussels I960.

Volume I, page 597 . 
Starting from the conception that reinsurance is a transformation of the distribution function of the risk business into another distribution function, which has a prescribed variance, this author proves, that stop loss reinsurance is the most efficient form of reinsurance, but concludes "whether it is also the cheapest form will depend on the loading".

If, however, the subject of optimal reinsurance is approached from an applied viewpoint, the matter of loadings should not be ignored the more so if we are considering stop loss reinsurance.

In this type of reinsurance contract the reinsurer is generally exposed to very considerable potential liabilities, that have a small probability to occur and consequently lead to modest net premiums.

It is obvious that in view of this, it is of particular importance for the reinsurer to establish an adequate contingency loading on the net premium.

In the approach outlined in the following pages we assume that the reinsurer consistently adds a constant fraction of the standard deviation of the net stop loss premium to this net premium.

\section{Model}

We assume a static and homogeneous risk portfolio i.e. no changes occur apart from claims and the single risks have identical basic probabilities.

The number of claims have a Poisson distribution (with mean $\lambda$ in the unit time period) and each claims has associated with it a positive random variable $x$, representing the claim size. The claim sizes are regarded as independent of the distribution of the number of claims, independent of each other and identically distributed with d.f. G. $(x)$.

A surplus reinsurance is in force for the excess over the retention limit $u$, which corresponds to a truncation of $G(x)$ at the point $u$

$$
G(x, u)=G(x)-H(x-u) G(x)+H(x-u) G(u)
$$

$H(x)$ stands for Heaviside's Unit Function, defined as

$$
\begin{aligned}
H(x) & =\mathrm{I} & & (x>0) \\
& =0 & & (x \leq 0)
\end{aligned}
$$


The characteristic function of $d F(x, u)$, which describes the behaviour of the risk business retained, becomes

$$
\exp \lambda\{(\varphi(s, u)-\mathrm{I})\} \quad \text { where } \varphi(s, u)=\int_{0}^{\infty} e^{i x s} d G(x, u)
$$

It is important to note that $u$ is regarded as a variable. The value assigned to it will have to satisfy some condition of optimality.

Employing for the moments of the conditional d.f. of the claim size the notation

$$
\mu_{u}(u)=\int_{0}^{u} x^{\nu} d G(x)+u^{\nu}\{\mathbf{I}-G(u)\} \quad v=\mathrm{I}, 2, \ldots
$$

the following transformed variable is introduced in $F(x, u)$

$$
\begin{aligned}
& x=\left\{\lambda \mu_{2}(u)\right\}^{1 / 2} z+\lambda \mu_{1}(u), \text { or } \\
& F\left[\left\{\lambda \mu_{2}(u)\right\}^{1 / 2} z+\lambda \mu_{1}(u)\right]=F_{0}(z, u)
\end{aligned}
$$

We expand the characteristic function for the transformed variable in ascending powers of $\lambda^{-1 / 2}$ and obtain the following, well known, series representation:

$$
\begin{gathered}
F_{0}(z, u)=\Phi(z)-\frac{\beta_{3}(u)}{3 ! \lambda^{1 / 2}} \Phi^{(3)}(z)+\frac{\beta_{4}(u)}{4 ! \lambda} \Phi^{(4)}(z)+\frac{\text { Io } \beta_{3}^{2}}{6 ! \lambda} \Phi^{(6)}(z)+\ldots \text { (3) } \\
\text { where } \Phi(z)=\frac{\mathrm{I}}{\sqrt{2 \pi}} \int_{-\infty}^{z} e^{-\frac{y^{2}}{2}} d y, \quad \Phi^{(n)}(z)=\frac{d^{n}}{d z^{n}} \Phi(z) \text { and } \beta \vee(u)=\frac{\mu_{u}(u)}{\left\{\left(\mu_{2}(u)\right\}^{\nu}\right.}
\end{gathered}
$$

It is to be stressed that $z$ is in fact a function of $u$.

\section{Asymptotic Expansions for the Net Stop Loss Premium and} its Standard Deviation.

Stop Loss reinsurance is understood to mean a reinsurance contract, covering the excess (if any) over a certain limit of the total of claims related to a fixed period of time.

The limit just mentioned will be expressed in relation to the mean claim $\lambda \mu_{1}(u)$ as the quotient between it and the mean claim. It will be named "priority" and denoted by the symbol $v$. 
From the definition the expressions for the net stop loss premium and its standard deviation are seen to be as follows

$$
\begin{gathered}
\pi(u, v)=\int_{\nu \lambda \mu,(u)}^{\infty}[x-v \lambda \mu(u)] d F(x, u) \\
\sigma^{2}(u, v)=\int_{\nu, \mu,(u)}^{\infty}\left[x-v \mu_{\mu_{1}}(u)\right]^{2} d F(x, u)-\pi^{2}(u, v)
\end{gathered}
$$

With the help of the transformation (2) we can write

$$
\begin{gathered}
\pi(u, v)=\left\{\lambda \mu_{2}(u)\right\}^{1 / 2} \int_{t_{u v}}^{\infty}\left(z-t_{u v}\right) d F_{0}(z, u) \\
\sigma^{2}(u, v)=\lambda \mu_{2}(u) \int_{t_{u v}}^{\infty}\left(z-t_{u v}\right)^{2} d F_{0}(z, u)-\pi^{2}(u, v)
\end{gathered}
$$

The indexes in $t_{u v}$ call attention to the fact that $t_{v u}$ depends on $u$ and $v$ as follows

$$
t_{u v}=\frac{(v-\mathrm{I}) \lambda \mu_{1}(u)}{\left\{\lambda \mu_{2}(u)\right\}^{1 / 2}}
$$

Without justifying whether this is permissible, we evaluate the integrals (6) and (7) by means of series representation (3) and obtain

$$
\begin{gathered}
\pi(u, v)=\left\{\lambda \mu_{2}(u)\right\}^{1 / 2}\left[-t_{u v}+t_{u v} \Phi\left(t_{u v}\right)+\Phi^{(1)}\left(t_{u v}\right)-\right. \\
\left.-\frac{\beta_{3}(u)}{3 ! \lambda^{1 / 2}} \Phi^{(2)}\left(t_{u v}\right)+\ldots\right] \\
\begin{aligned}
\sigma^{2}(u, v)=\lambda \mu_{2}(u)\left[\left(\mathrm{I}+t_{u v}^{2}\right)\{\mathrm{I}\right. & \left.-\Phi\left(t_{u v}\right)\right\}-t_{u v} \Phi^{(1)}\left(t_{u v}\right)+ \\
& \left.+\frac{2 \beta_{3}(u)}{3 ! \lambda^{1 / 2}} \Phi^{(1)}\left(t_{u v}\right)+\ldots\right]-\pi^{2}(u, v)
\end{aligned}
\end{gathered}
$$

If $\lambda$ is assumed to be large (e.g. greater than Ioo) we may reasonable neglect the terms involving $\lambda^{-1 / 2}$ and its higher powers.

For the remainder we write for short

$$
\begin{aligned}
P\left(t_{u v}\right) & =-t_{u v}+t_{u v} \Phi\left(t_{u v}\right)+\Phi^{(1)}\left(t_{u v}\right) \\
S^{2}\left(t_{u v}\right) & =\left(\mathrm{I}+t_{u v}^{2}\left\{\left(\mathrm{I}-\Phi\left(t_{u v}\right)\right\}-t_{u v} \Phi^{(1)}\left(t_{u v}\right)-P^{2}\left(t_{u v}\right)\right.\right. \\
& =\mathrm{I}-\Phi\left(t_{u v}\right)-t_{u v} P\left(t_{u v}\right)-P^{2}\left(t_{u v}\right)
\end{aligned}
$$


And finally

$$
\begin{aligned}
\pi(u, v) & =\left\{\left(\lambda \mu_{2}(u)\right\}^{1 / 2} P\left(t_{u v}\right)\right. \\
\sigma^{2}(u, v) & =\lambda \mu_{2}(u) S^{2}\left(t_{u v}\right)
\end{aligned}
$$

The approximations (I3) and (I4) will be used in the sequel.

4. Effect of Stop Loss Reinsurance on the Variance of a Risk Portfolio.

The conclusion of a stop loss reinsurance will have a lowering effect on the variance of the risk born by the insurer.

As this variance is adopted as a proper measure of the stability, it is useful to establish a relation between the variance before the introduction of a stop loss and after, in order to have a means for adapting the characteristics of the stop loss to the desired amount of reduction in the variance.

First let it be mentioned that a stop loss reinsurance will affect the mean claim for own account as follows

$$
\begin{aligned}
& E(c)=\int_{0}^{\nu \lambda \mu_{1}(u)} x d F(x, u)+v \lambda \mu,(u) \int^{\infty} d \overline{\mu_{1}(u)} \\
& E(c)=\lambda \mu_{1}(u)-\pi(u, v)
\end{aligned}
$$

As was to be expected the mean claim decreases by the amount of the net stop loss premium.

Using eqn. 15 , the variance, if a stop loss reinsurance for a net premium $\pi(u, v)$ is effected, is seen to be

$$
\begin{aligned}
E\{c-E(c)\}^{2}= & \int_{0}^{v \lambda \mu_{1}(u)}\left[x-\left\{\lambda \mu_{1}(u)-\pi(u, v)\right\}\right]^{2} d F(x, u)+ \\
& +\left[\nu \lambda \mu_{1}(u)-\left\{\left(\lambda \mu_{1}(u)-\pi(u, v)\right\}\right]^{2} \int_{v \lambda \mu_{1}(u)}^{\infty} d F(x, u)\right.
\end{aligned}
$$

Writing this in full and suitably rearranging the terms, it follows that

$$
E\left\{(c-E(c)\}^{2}=\lambda \mu_{2}(u)\left\{S^{2}\left(t_{u v}\right)+2 \Phi\left(t_{u v}\right)-\mathrm{I}\right\}\right.
$$

For the third factor in $(\mathrm{I} 7)$, we use the abbreviation

$$
\delta^{2}\left(t_{u v}\right)=S^{2}\left(t_{u v}\right)+2 \Phi\left(t_{u v}\right)-\mathrm{I}
$$


We now apply eqn. I 7 to an insurance portfolio for which only a surplus reinsurance is in force, with retention limit $u_{0}$. The choice of $u_{0}$. corresponds to a variance $\lambda \mu_{2}\left(u_{0}\right)$.

Subsequently a stop loss is introduced in addition to the surplus reinsurance, and we require the variance of the retained risk not to deviate from the initial value.

Assuming the parameter $\lambda$ not be subject to any changes, it is obvious from $(\mathrm{r} 7)$ that all pairs $u$ and $v$, which satisfy

$$
\mu_{2}\left(u_{0}\right)=\mu_{2}(u) \delta^{2}\left(t_{u v}\right)
$$

lead to a variance $\lambda \mu_{2}\left(u_{0}\right)$, for the risk retained by the insurer.

Next we examine the second factor in eqn. Ig.

Considering eqn's $I 2$ and $\mathbf{I} 8$, it appears that, if $t_{u v}=0$

$$
\delta^{2}(0)=1 / 2-\left\{\Phi^{(1)}(0)\right\}^{2} \sim 0.34
$$

For $t_{u v}$ running from zero to infinity, it is easily seen that $\delta^{2}\left(t_{u v}\right)$ is a monotonically increasing function, attaining unity at infinity.

Thus it appears that a value of approximately 0.34 is the minimum of $\delta^{2}\left(t_{u v}\right)$ for positive argument.

This implies, that a stop loss reinsurance cannot reduce the variance of a given group of risks, to less than about $I / 3$ of the amount of variance the risks would have if no stop loss were in force.

Hence, introduction of stop loss reinsurance, does not allow the increase of the retention limit beyond a certain maximum corresponding to a variance which is about three times the initial variance.

According to eqn. 8, for $t_{u v}=0$, the variable $v$ will have the value unity. This means that, if the maximal reduction of variance has been achieved, the reinsurer pays all claims in excess of the mean claim amount and consequently the maximal extent of stop loss cover has been obtained by the insurer.

\section{Minimization of the Total Reinsurance Cost.}

The types of reinsurance considered here are surplus- and stop loss reinsurance. 
The possibility of e.g. quota share cession will be ignored.

To find the minimum cost, we will have to assign such values to $u$ and $v$ which satisfy the minimum of the following cost function

$$
C(u, v)=\gamma \lambda \int_{u}^{\infty}(x-u) d G(x)+\varepsilon\left\{\left(\lambda \mu_{2}(u)\right\}^{1 / 2} S\left(t_{u v}\right)\right.
$$

The first term in the second member of (2I) represents the total profit margin $\gamma$, which is ceded if the retention is $u$.

The second term is the loading on the net stop loss premium, for which we assume the reinsurer to charge a fraction $\varepsilon(<\mathrm{I})$ of the standard deviation of the net stop loss premium.

It is noticeable that the first term is a decreasing function of $u$ while the second increases with increasing $u$.

This makes it reasonable to expect $(2 \mathrm{I})$ to have a minimum value under certain conditions.

The minimum will be, however, subject to the constraint imposed by eqn. I9, since the variance of the risk retained, should have a constant value throughout.

We now obtain the minimum by equating to zero, the differential coefficients with respect to $u$ and $v$, of the following equation

$$
\begin{aligned}
C(u, v)=\delta \lambda \int^{\infty}(x-u) d G(x) & +\varepsilon\left\{\lambda \mu_{2}(u)\right\}^{1 / 2} S\left(t_{u v}\right)+ \\
+ & L\left[\left\{\lambda_{\mu_{2}}(u)\right\}^{1 / 2} \delta\left(t_{u v}\right)-\left\{\lambda_{\mu_{2}}(u)\right\}^{1 / 2}\right]
\end{aligned}
$$

where we have introduced the Lagrangian multiplier $L$.

Differentiating

$$
\begin{aligned}
\frac{\partial C(u, v)}{\partial u}= & -\gamma \lambda[\mathbf{I}-G(u)]+\frac{\lambda u}{\left\{\lambda \mu_{2}(u)\right\}^{1 / 2}}[\mathbf{I}-G(u)]\left[\varepsilon S\left(t_{u v}\right)+\right. \\
& \left.+L \delta\left(t_{u v}\right)\right]+\frac{\partial t_{u v}}{\partial u}\left\{\lambda \mu_{2}(u)\right\}^{1 / 2}\left[\varepsilon S^{\prime}\left(t_{u v}\right)+L \delta^{\prime}\left(t_{u v}\right)\right]=0 \\
& \frac{\partial C(u, v)}{\partial v}=\frac{\partial t_{u v}}{\partial v}\left\{\lambda \mu_{2}(u)\right\}^{1 / 2}\left[\varepsilon S^{\prime}\left(t_{u v}\right)+L \delta^{\prime}\left(t_{u v}\right)\right]=0
\end{aligned}
$$

Eliminating $L$ from eqn's 23 and 24 , we obtain 


$$
\frac{\gamma\left\{\lambda \mu_{2}(u)\right\}^{1 / 2}}{u}=\left[\varepsilon\left[S\left(t_{u v}\right)-\delta\left(t_{u v}\right) \frac{S^{\prime}\left(t_{u v}\right)}{\delta^{\prime}\left(t_{u v}\right)}\right]\right.
$$

The differential coefficients, occurring in the right hand member, can be found by differentiating (I2) and (18) with respect to $t_{u v}$. We have

$$
\begin{aligned}
S^{\prime}\left(t_{u v}\right) & =-\frac{P\left(t_{u v}\right) \Phi\left(t_{u v}\right)}{S\left(t_{u v}\right)} \\
\delta^{\prime}\left(t_{u v}\right) & =\frac{\left[t_{u v}+P\left(t_{u v}\right)\right]\left[\mathrm{I}-\Phi\left(t_{u v}\right)\right]}{\delta\left(t_{u v}\right)}
\end{aligned}
$$

Substitution in eqn. 25 of (26) and (27) and making use of (I9) leads to

$$
\begin{aligned}
& \frac{\gamma\left\{\lambda \mu_{2}\left(u_{0}\right)\right\}^{1 / 2}}{\varepsilon u}=\frac{\delta\left(t_{u v}\right)}{S\left(t_{u v}\right)}\left[\mathrm{I}-2 \Phi\left(t_{u v}\right)+\right. \\
& \left.\quad+\frac{\Phi^{(1)}\left(t_{u v}\right) \delta^{2}\left(t_{u v}\right)}{\left\{t_{u v} \Phi\left(t_{u v}\right)+\Phi^{(1)}\left(t_{u v}\right)\right\}\left\{\mathrm{I}-\Phi\left(t_{u v}\right)\right\}}\right]
\end{aligned}
$$

The right hand member of eqn. 28 will be denoted by $\Psi^{*}\left(t_{u v}\right)$. Although it looks difficult to handle, tabulation for a suitable range of $t_{u v}$ can be easily performed, as $\Psi\left(t_{u v}\right)$ depends on $t_{u v}$ exclusively and not on the parameters of the case in hand. These parameters are all collected in the lefthand member of eqn. 28.

Examination of $\Psi^{\prime}\left(t_{u v}\right)$ reveals that $\Psi^{\prime}(0)=0.68$ and that for positive $t_{u v}$, it converges monotonically towards infinity as $t_{u v}$ goes to infinity i.e. no zero's occur for positive values of $t_{u v}$.

From the latter fact we infer that a solution for the optimal retention must always be finite, in view of the fact that the reciprocal of $u$ occurs in the lefthand side of eqn. 28 .

This eliminates the possibility that a minimization of reinsurance cost, could ever be obtained purely by means of a stop loss contract, as this would require inclusion of infinity in the admissible range of $u$.

The possibility that exclusively a surplus reinsurance will provide an optimal solution can be excluded as well if we consider that then the optimal retention will take the value $u_{0}$. 
By virtue of eqn. I9 this corresponds to infinite $t_{u v}$, which in turn will make the righthand member of eqn. 28 infinitely great.

This leads, however, to a contradiction, since the lefthand member of this eqn. remains finite since $u$ is greater than zero.

Hence, the conclusion can be drawn that the cheapest reinsurance will always be a combination of surplus- and stop loss if we limit the choice between these two types.

It should be added, however, that for very large $\lambda, u$ will turn out that large, that for practical purposes, we can speak of a pure stop loss reinsurance.

As a rule very large $\lambda$ do not occur in such cases as are current in the reinsurance market. It so happens that $\lambda$ is commonly seen to have values below Iooo.

If, on the other hand $\lambda$ is very small, say below Ioo, eqn's 19 and 28 will become rather inaccurate as a result of the approximation error we have introduced by neglecting terms involving the successive powers of $\lambda^{-1 / 2}$. However, for such small number of expected claims, the reduction in reinsurance cost obtainable by finding the minimum is hardly of practical interest.

The solution of $u$ and $v$ in practical cases proceeds from eqn's 8 , I9 and 28 . The functions $\Psi\left(t_{u v}\right)$ and $\delta^{2}\left(t_{u v}\right)$ that depend on $t_{u v}$ only, can be tabulated.

For this purpose the range from o to I is sufficient.

Solutions for $u$ and $t_{u v}$ are obtained first by trial and error from eqn's 19 and 28 . From eqn. 8 and the solution of $u$ and $t_{u v}$ the corresponding $v$ can be found.

\section{Numerical Example.}

In the table below the sequences of ceded profit are shown if the initial retention $u_{0}$ is gradually increased. For each $u$ the priority of the stop loss reinsurance has been chosen so as to bring the variance down to the level of variance in the initial stage.

The conditional density function of the claim size is $\frac{\alpha^{3} x^{2} e^{-\alpha x}}{2 !}$, $\alpha$ having the value $0.000,06$.

A profit loading of $5 \%$ is assumed for the surplus reinsurance and a loading of $50 \%$ of its standard deviation has been added consistently to the net stop loss premium. 


\begin{tabular}{|c|c|c|c|c|c|c|}
\hline$\lambda$ & \multicolumn{2}{|c|}{400} & \multicolumn{2}{|c|}{625} & \multicolumn{2}{|c|}{795} \\
\hline$u$ & IOO $v$ & $\begin{array}{l}\text { ceded } \\
\text { profit }\end{array}$ & I $00 v$ & $\begin{array}{l}\text { ceded } \\
\text { profit }\end{array}$ & IOO $v$ & $\begin{array}{l}\text { ceded } \\
\text { profit }\end{array}$ \\
\hline I I 5,000 & $\infty$ & 342,500 & $\infty$ & 535,000 & $\infty$ & $68 \mathrm{I}, 000$ \\
\hline I 35,000 & 105.9 & 357,000 & 104.7 & 530,500 & 104.2 & 669,000 \\
\hline I 50,000 & 104.4 & $35^{0}, 5^{\circ 0}$ & 103.5 & 508,500 & I03. I & 624,000 \\
\hline I 75,000 & 102.9 & 340,000 & 102.4 & 475,500 & IO2.I & 573,000 \\
\hline 200,000 & IO2. I & 333,500 & Iо1. 6 & 453,000 & IOr. 5 & 537,000 \\
\hline 225,000 & IOI. 5 & 328,000 & IOI. 2 & 436,000 & IOI.I & 510,000 \\
\hline 250,000 & IOI.I & 326,500 & 100,8 & 426,000 & I00.8 & 493,000 \\
\hline 275,000 & 100.8 & 325,000 & 100.7 & 418,000 & I00.6 & 480,500 \\
\hline 300,000 & 100.6 & 326,500 & I00. 5 & 417,000 & 100.4 & 476,500 \\
\hline 325,000 & 100.4 & 328,500 & 100.3 & 416.000 & 100. 3 & 473,500 \\
\hline 350,000 & 100.3 & 329,000 & 100.3 & $4 I 5 \cdot 500$ & 100.2 & 471,500 \\
\hline 375, ,ooo & 100.3 & $3^{29}, 5^{\circ 0}$ & 100.2 & $41_{4}, 500$ & IOO. 2 & 469,500 \\
\hline 400,000 & 100.2 & 330,500 & 100.2 & 4 I 5,000 & IOO. 2 & 469,000 \\
\hline 450,000 & IOO.I & 332,500 & IOO.I & 416,000 & IOO. I & 470,000 \\
\hline 500.000 & IOO.I & 333,000 & IOO.I & $4 I 6,500$ & IOO.I & $47^{\circ}, 500$ \\
\hline$\infty$ & 100 & 337,000 & 100 & 421,500 & IOO & 475,000 \\
\hline
\end{tabular}

